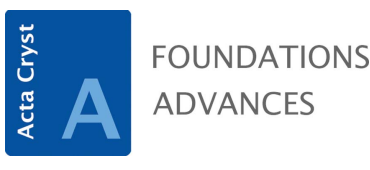

ISSN 2053-2733

\section{Numerical Problems in Crystallography. By M. A. Wahab. Springer, 2021. Hardcover, pp. xv + 387. ISBN 9789811597534. Price EUR 83.19.}

\author{
Massimo Nespolo*
}

Université de Lorraine, CNRS, CRM2, Nancy, France. *Correspondence e-mail: massimo.nespolo@univ-lorraine.fr

The idea of writing a book presenting a series of concrete, simple computational situations possibly occurring in the daily life of a crystallographer, rather than another textbook saying more or less what has been explained countless number of times, is certainly welcome. The public reading such a manual is expected to already know the basics; the content should therefore include only a minimal number of definitions, as a reminder, and proceed straight to the examples and exercises. This is more or less the approach adopted in Numerical Problems in Crystallography. Unfortunately, the result is not a success. This is not only because many topics are inexplicably left out (e.g. the metric tensor and any notion of space-group symmetry are completely absent) but also because of the large number of inconsistencies and errors.

The book is organized into nine chapters, six of which are about unit cells, each ending with a list of multiple-choice questions (four choices for each question), with the answers at the end, preceded by tables of units and physical quantities, of which only a small minority are relevant to the text.

The first chapter (40 pp.) has the title Unit Cell Composition but is mainly about Bravais lattices, with some considerations about sphere packings at the end. In this chapter we 'learn' about eight crystal systems and sixteen types of Bravais lattices. Yes, you have read it correctly. Two new types of lattice (and one more crystal system) are proposed. Let us see where this illogical situation comes from. The details are not given here (remember, this is not a textbook, but a book of exercises), but we find the answer in Wahab \& Wahab (2015). As I have already explained (Nespolo, 2019), this incredible confusion partly comes from misunderstanding the fundamental difference between a lattice and a structure. The author misinterprets the hexagonal close-packed (h.c.p) structure as a lattice, so that they appear to have discovered an h.c.p. lattice. The second ghost is a hypothetical 'rhombohedral close packed lattice (RCP)', with cell parameters $a=b=c, \alpha=\beta=\gamma=60^{\circ}$. Every student of crystallography knows that this is simply the primitive cell of a face-centred cubic lattice. But let us proceed. In two dimensions, we find only four types of lattices, instead of five (p. 16), which however become five two pages later. This probably comes from a confusion between the notions of lattice and of crystal system, one of the many confusions in this book. The list of point groups (an undefined term) assigned to each lattice is also wrong. For the oblique lattice, we have $\{1\}$ and $\{m\}$ (the brackets are not used in the book): how an oblique lattice can possess a mirror line remains unexplained (and is, of course, unexplainable). Point group $\{2\}$ is assigned to the rectangular lattices; the places of $\{2\}$ and $\{m\}$ should be exchanged. The restrictions on cell parameters are also wrong, because metric specializations are ignored. This is an unfortunately common mistake, as I have already pointed out (Nespolo, 2015). The exercises involve the calculation of the length of translation vectors, some changes of basis, computation of the volumes of unit cells and analysis of possible centring of unit cells. All without metric tensor, which would have made these exercises trivially simple. The last part deals with simple examples of close packing of spheres.

The second chapter (54 pp.) is about Unit Cell Construction and deals with WignerSeitz cells, Brillouin zones and reciprocal lattices. Even if one ignores awkward expressions like 'trigonal lattice' and 'trigonal unit cell', which also appear in some very well known textbooks, here we find nonsensical phrases like ' $\mathrm{CsCl}$ lattice' and ' $\mathrm{NaCl}$ lattice'. The consequences of not understanding the difference between a lattice and a structure are severe. For example, the lattice of $\mathrm{CsCl}$ being $c P$, its Wigner-Seitz cell is a cube. But in 
this book, 27 unit cells are used to eventually obtain a huge octahedron (Fig. 2.9). (Note: vectors between Cs atoms are used to get the Wigner-Seitz cell, which should be defined with respect to a lattice.) The first exercises are on the WignerSeitz cells and are all about two-dimensional lattices, but suddenly a third dimension is used. The other exercises are about the reciprocal lattice of a given direct lattice and the corresponding (first) Brillouin zone; the absence of the metric tensor obliges the reader to perform unnecessary mental gymnastics.

Chapter 3 (42 pp.) is about Unit Cell Calculations. It deals with fractional coordinates, distances and angles in the unit cell, formula units, and packing efficiency. The list of incorrect or awkward expressions is also long in this chapter.

The statement 'a twofold rotation along the z-axis will change the fractional coordinates $(x, y, z)$ into $(\bar{x}, \bar{y}, z)$, a mirror plane perpendicular to the $\mathrm{z}$-axis will change $(x, y, z)$ into $(x, y, \bar{z})^{\prime}$ is correct only if one specifies that the corresponding symmetry elements pass through the origin, which is far from being true in general.

The sentence 'the determination of fractional coordinates in higher symmetry crystal systems becomes increasingly cumbersome' is surprising, to say the least, considering that most examples in this book are limited to high-symmetry cases.

How 'a point' can be 'consisting of asymmetric group of atom/atoms' remains a mystery.

The 'fractional coordinates of other equivalent positions' are said to be obtained 'under a given point group'. Are there then no translations in a crystal?

Ignoring the fundamental concept of order (used much later in the book and never defined) leads to impossible sentences requiring mental contortions, such as 'Total number of equivalent positions for a given point group is the product of number of equivalent points of each independent symmetry element/operation with the number of independent symmetry elements/operations.'

Table 3.1 is expected to present the matrix representation of symmetry operations and the corresponding coordinate triplets for the ten types of point groups in two-dimensional space. For each of them, however, only one matrix is presented, even for non-cyclic groups. And the indication 'parallel to z-axis' is nonsense: in a two-dimensional space there is no $z$ axis (meaning a third independent axis), by definition.

The exercises proposed are elementary but become unnecessarily time-consuming: the use of the metric tensor would have made most of them unnecessary, and the others trivial. For example, the angle between two directions in Table 3.3 is given in the form of long and complicated formulas, and only for higher-symmetry cases; a simple formula with the metric tensor would have made this table useless. In fact, the metric tensor does show up at p. 109, to compute the volume of a unit cell, but is not even mentioned as such.

Section 3.5 is perturbed by the usual confusion between lattice and structure, with an endless swap between 'atoms' and 'lattice points'. Expert readers will bump their heads against a wall; beginners will be lost.

In the first exercise of the section on 'packing efficiency' ( $p$. 121) we read about a '2-D arrangement of identical spheres'. How a sphere can show up in a two-dimensional space is one of the many mysteries of this book. And in the fourth exercise we even read about ' $\mathrm{NaCl}$ molecules'. Cleary, crystal chemistry is disregarded (we find other examples in Chapter 8: a ' $\mathrm{Cu}_{3} \mathrm{Au}$ molecule' on p. 311 and a ' $\mathrm{CaF}_{2}$ molecule' on p. 325 ).

Chapter 4 (42 pp.) has the title Unit Cell Representations of Miller Indices and is another battlefield from the beginning, where we read about Miller indices of atomic sites and of directions. Weber indices and Bravais-Miller indices are mixed up (p. 149). Miller indices of planes are sometimes reduced to non-prime integers, also when they should not be (Nespolo, 2015). Direction indices are always given as integers, also when they should not be (Nespolo, 2017). The confusion between lattice nodes and atomic positions shows up again and again. This leads to consideration of lattice sites that do not exist, through which lattice directions are expected to pass, such as $\frac{1}{2}, 1,0$ (p. 143) or $\frac{1}{3}, \frac{1}{3}, \frac{2}{3}$ (p. 148). ${ }^{\mathbf{1}}$ The number of equivalent lattice directions is systematically wrong, namely doubled, because $[u v w]$ and $[\bar{u} \bar{v} \bar{w}]$ are considered as different directions. The common mistake of imposing different values of cell parameters along non-equivalent directions is systematically present in the exercises (a reminder for the lessattentive reader: metric specialization does not increase the symmetry of the crystal structure!). Apart from the large number of mistakes and inconsistencies, the exercises are essentially the application of formulas which, in most cases, could be avoided by the use of the metric tensor.

Chapter 5 (29 pp.) is about Unit Cell Transformations and presents a series of examples of change of coordinate system for orthorhombic or higher-symmetry crystals (it is not surprising that, by ignoring the metric tensor, examples of lower-symmetry crystals had to be excluded). The usual confusion between trigonal and rhombohedral makes this even more disturbing to read.

Chapter 6 (56 pp.) deals with Unit Cell Symmetries and Their Representations but is devoted to matrix representation of symmetry operations, so it is puzzling why the words 'unit cell' appear in the title. After the classical geometrical introduction of the crystallographic restriction on the order of rotation we are told (p. 214) that in a monoclinic crystal there is a twofold axis (taken along $c$ - why not?), a 'center of symmetry' and 'two mirror planes intersecting at $90^{\circ}$ whose axis is parallel to the c-axis', so that the total would be '1-diad +2 surface planes +1 center of symmetry $=4$ '. Combine them and you get $\mathrm{mmm}$, which is of order 8 , not 4 , and is orthorhombic, not monoclinic. The same nonsense is repeated in Example 6 (p. 226). But we are not done yet. In a tetragonal unit cell one would have '4-diads +1 -tetrad +7 [sic] surface planes +1 center of symmetry $=13^{\prime}$. By the way, the identity is

\footnotetext{
${ }^{1}$ Lattice nodes with these coordinates can of course exist if one chooses a nonconventional unit cell with unusual centrings not among those for which a letter has been assigned ( $P, A, B, C, I, F, R, H, D)$. This is clearly not the case here.
} 
never mentioned. After a series of simple calculations to obtain the matrices representing some symmetry operations, we come to a section devoted to point groups, where the confusion between symmetry elements and symmetry operations is widespread, but is not the most serious defect. The term 'independent' is erroneously used, because even in a cyclic group the operations are described as 'independent'. Example 1 for point group 3: three independent operations; Example 2 for point group $2 / m$, four independent operations; Example 3 for point group $m m 2$, again four independent operations. Then at p. 246 we are told that space groups are finite groups. The infinitely many translations, and the infinitely many operations they generate, must have been forgotten. The partition of symmetry operations into (conjugacy) classes is done in an intuitive and non-exhaustive way because the use of conjugacy relations is 'too elaborate $[\ldots]$ in high symmetry systems' [sic].

Chapter 7 (35 pp.) deals with Diffraction of Waves and Particles by Crystal. It begins with a short discussion about the production of X-rays, where we read that 'The characteristic $\mathrm{X}$-rays $\left(\mathrm{K}_{\alpha}, \mathrm{K}_{\beta}\right.$, etc. $)$ are produced when the bombarded electrons eject some of the orbital electrons of the target atom and the corresponding vacancies are filled by the less energetic outer shell electrons'. This implies that outer-shell electrons should gain energy following the transition, instead of losing it. Then follows a long and tedious list of examples illustrating how to calculate the wavelength of a given transition, and even one in which Planck's constant is calculated, before arriving at a section about diffraction. Bragg's law is stated but not demonstrated (after all, this is not a textbook but a book of exercises). This short section ends with the enunciation of Laue's equations followed by a series of examples in which Bragg's law is applied. The Bragg angle for 110 diffraction by rock salt is computed in Example 9 on p. 278; why choose a reflection that is absent because of the integral reflection conditions (the conventional unit cell of rock salt is $c F$ )? Moreover, the result is wrong, because the calculation is performed as $\theta=\sin ^{-1}(\lambda / d)$ instead of $\theta=\sin ^{-1}(\lambda / 2 d)$ (the same problem is found in the following example, No. 10). Again, in Exercise 13 (p. 280), for the same crystal, the firstorder diffraction from planes (100), (110) and (111) are computed; the first two reflections are absent because of the integral reflection conditions. Next comes a section on absorption of X-rays, with a few examples of calculations of the attenuation of the X-ray beam. Example 3 uses wavelengths of $0.064 \AA$ and $0.098 \AA$, which are in the region of gamma rays, not $\mathrm{X}$-rays. The last, short, section is on 'Other diffraction methods': neutron and electron diffraction are 'explained' in four lines each, followed by examples to compute wavelengths: nothing is said about diffraction.

Chapter 8 (38 pp.) presents Structure Factor Calculations and applies formulas that are not demonstrated. Example 1 computes the phase angle for the reflection 000; Example 2 is about the phase angle of an atom at the origin (how can one have a phase angle of an atom?). Does one really need these types of examples? Things get hard when simple structures are considered. One may wonder how it is possible to compute structure factors without any notion of space-group symmetry, a topic outside the scope of this book. One can of course use the fractional coordinates of all the atoms in the unit cell as if the space group were of type $P 1$; but one should use the correct coordinates. Example 10 illustrates how far one can go wrong. This example is about an h.c.p. structure. Without any space-group symmetry, the coordinates of all atoms should be given in $P 1$. Now, an h.c.p. structure has two atoms in the unit cell of $P 6_{3} / m m c$ in Wyckoff position $2 c$, coordinates $\frac{1}{3}, \frac{2}{3}, \frac{1}{4}$ and $\frac{2}{3}, \frac{1}{3}, \frac{3}{4}$. The coordinates given in the example are $0,0,0$ and $\frac{1}{3}, \frac{2}{3}, \frac{1}{2}$, i.e. with an origin shift of $-\frac{1}{3},-\frac{2}{3},-\frac{1}{4}$. That something is wrong should be evident from the fact that the calculation results in a complex structure factor despite the fact that the structure is centrosymmetric. In this description, the inversion centre is at $\frac{1}{6}, \frac{1}{3}, \frac{1}{4}$; it is not suprising that it is missed. To overcome this difficulty the book seeks help from quantum mechanics, while the problem comes just from the wrong coordinates. Another significant error is found in Example 14, where a hypothetical tetragonal crystal is described having four atoms in the unit cell, two of which differ from the other two by a $c / 2$ translation; there are actually only two atoms in the unit cell, whose $c$ period has to be halved. The chapter ends with an statement about the impossibility of determining the presence or absence of an inversion centre from diffraction data. It seems the author has not heard of resonant scattering.

Chapter 9 (45 pp.) has the inappropriate title Determination of Crystal Structure Parameters. Structural parameters are not the topic of this chapter, which just involves some calculations of interplanar spacings and distances of peaks in powder diffraction patterns, and unit-cell parameters, i.e. essentially a continuation of what has already been presented in the previous chapter. The content goes back to the very early history of X-ray crystallography: 'Crystal structure determinations in general are not easy and straightforward. They are normally done by trial and error method' (p. 337). Experimental methods discussed here are powder diffraction, the Laue method and the rotation/oscillation method. Even Buerger's classic textbook (Buerger, 1942) is more modern and advanced.

A non-exhaustive list of problems that do not need detailed discussion follows.

Some terms are used without or before being defined (e.g. point group, crystal system).

Laue indices are given between parentheses, which should be reserved for Miller indices.

The difference between a lattice, a crystal and a crystal system is completely unclear, as shown by the frequent occurrence of expressions like 'a simple orthorhombic crystal system'. Adjectives like 'simple' do not apply here: a crystal system is a category, it is neither simple nor centred nor complex.

'Body centering' for a two-dimensional lattice (p. 21).

'Tetrahedral' instead of 'tetragonal' (p. 24).

$6 / \mathrm{mmm}$ and $m \overline{3} m$ (with the overline missing) are said to have no proper supergroup; which is of course wrong unless the restriction 'crystallographic' is added (p. 246). 
'In general, every group has two trivial subgroups, the identity element and the group itself.' An element is not a group (p. 246).

A simplified version of the group-subgroup tree for point groups shows a list of empty horizontal ovals. Their significance is not explained (p. 247).

'When a $\mathrm{Cu}$ crystal is subjected to an X-ray beam the firstorder (111) plane appears at an angle of $21.7^{\circ}$ (p. 275). Do we see a plane in a diffraction pattern?

Diffractometer is called 'spectrometer' (p. 280).

The atomic scattering (form) factor is called the 'atomic structure factor' (p. 300).

Systematic absences are called 'extinctions' (p. 307). Extinction is a completely different phenomenon. Although this is quite a common mistake in many older textbooks, we do not expect to still find it today.

'A diamond structure is $[\ldots]$ built up from two interpenetrating lattices' (p. 313). The same is found when discussing the $\mathrm{ZnS}$ structure (p. 320), which is described as 'two interpenetrating lattices' of $\mathrm{Zn}$ and of $\mathrm{S}$ (as if a lattice had anything to do with the atoms in the unit cell).

Considering the number of mistakes present in this book, one question remains to be answered: How is it possible for a publisher to agree to publish a book with such a high level of errors? The answer is most likely in the publishing model. Unlike a research paper, a manuscript submitted for a book seldom undergoes a review process. The opinion of some experts is often asked about the proposed table of contents, but nobody can guess what is really contained in the text. The result is that books like this go to press and result in serious harm to the reputation of the publisher.

\section{References}

Buerger, M. J. (1942). X-ray Crystallography. New York: John Wiley and Sons.

Nespolo, M. (2015). J. Appl. Cryst. 48, 1290-1298.

Nespolo, M. (2017). J. Appl. Cryst. 50, 1541-1544.

Nespolo, M. (2019). J. Appl. Cryst. 52, 451-456.

Wahab, M. A. \& Wahab, K. M. (2015). ISST J. Appl. Phys. 6, 1-10. 